\title{
هقارنة بعض طرائق التعويض الأحادي للبيانات المفقودة لدالة الكثافة الاحتمالية للتوزيع الطبيعي ثنائي المتغيرات
}

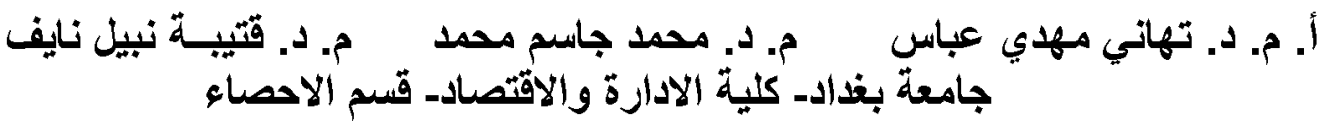

في هذا البحث تم اقتراح طريقة جيدة للتويض عن القيم المفقودة في حالة دالة التوزيع الطبيعي

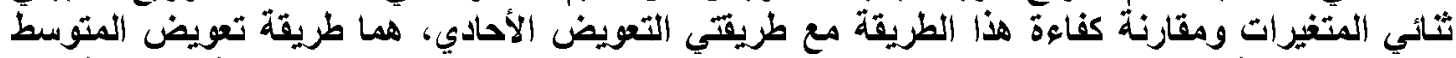

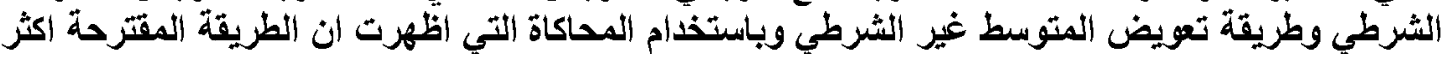

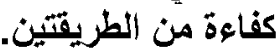

Abstract:

In this paper we suggest new method to estimate the missing data in bivariate normal distribution and compare it with Single Imputation method (Unconditional mean and Conditional mean) by using simulation.

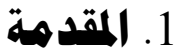

من المشاكل التي تواجه الباحثين أو القائمين على اتخاذ القرار هو وجود مشكلة المشاهدات المفقودة

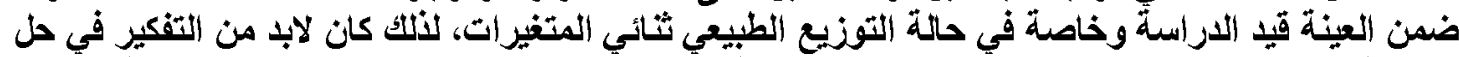

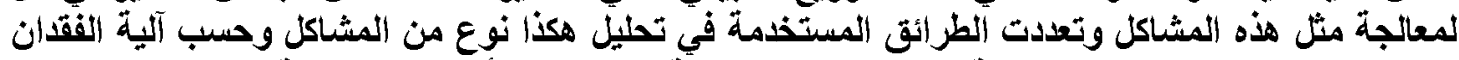

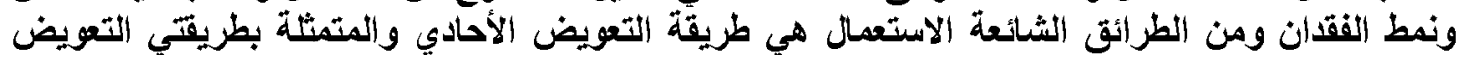

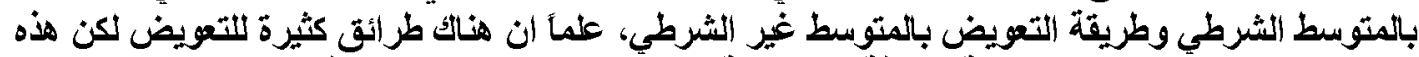

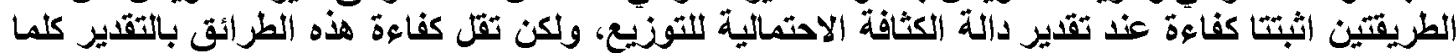

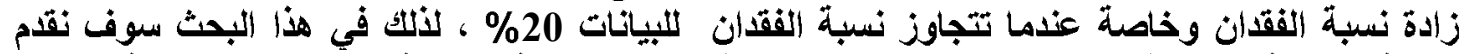

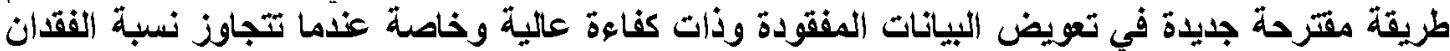

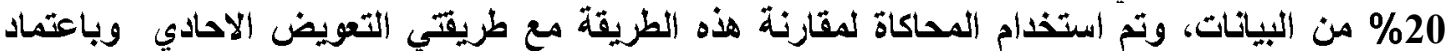
معيار متوسط مريعات الخطأ.

$$
\begin{aligned}
& \text { 2. الجانب النظري }
\end{aligned}
$$

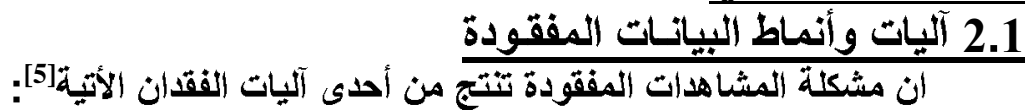

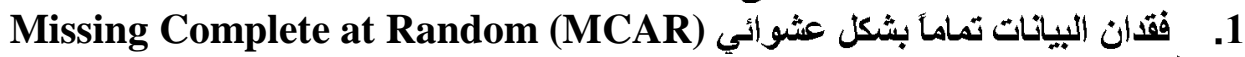

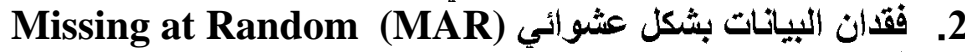

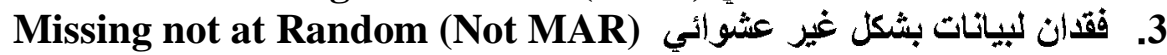


أن لأي آلية من الآليات المبينة أثقا مسببات في الظهور وعلى ضؤها تظهر مشكلة البيانات المفقودة علرئات

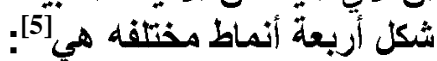

1

2.

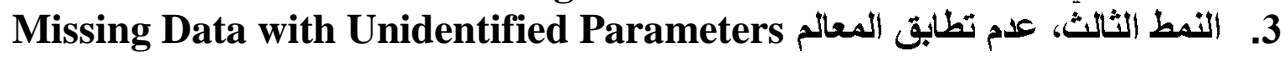

4. النمط العام

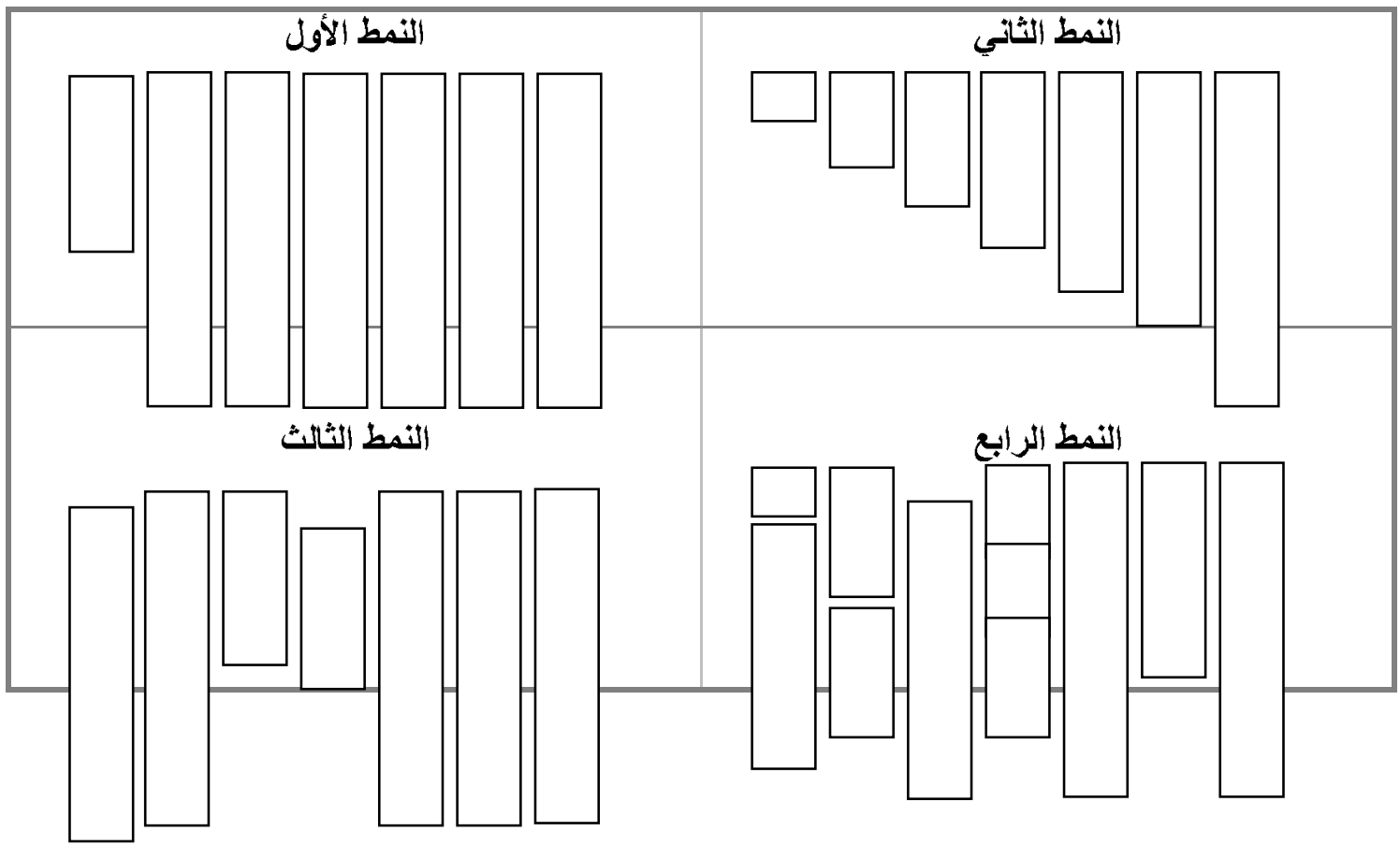

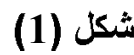

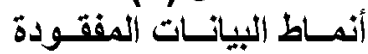

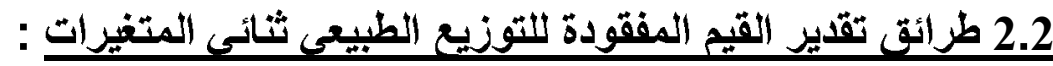

اذأ كان لدينا متجه المتغيرات

$\underline{\mathrm{x}}^{\prime}=\left[\mathrm{x}_{1}, \mathrm{x}_{2}, \ldots . ., \mathrm{x}_{\mathrm{p}}\right]$

$f(\underline{x})=\frac{1}{(2 \pi)^{1 / 2 p}|\Sigma|^{1 / 2}} \exp \left[-\frac{1}{2}(\underline{x}-\underline{\mu})^{\prime} \Sigma^{-1}(\underline{x}-\underline{\mu})\right.$

وأن

$\underline{\mu^{\prime}}=\left[\mu_{1}, \mu_{2}\right]$

$\Sigma=\left[\begin{array}{ll}\sigma_{11} & \sigma_{12} \\ \sigma_{21} & \sigma_{22}\end{array}\right]$ 
الطبيعي ثنائي المتغيرات

حيث

ب

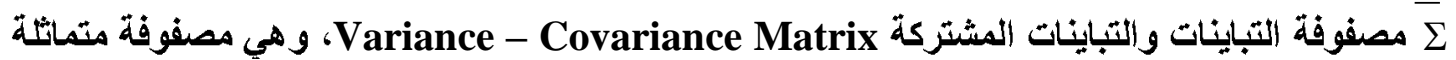

.Symmetric

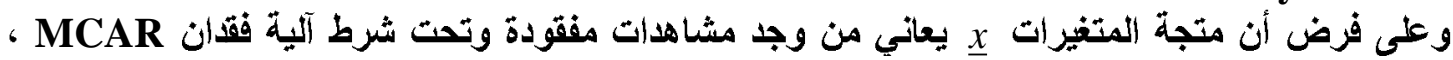

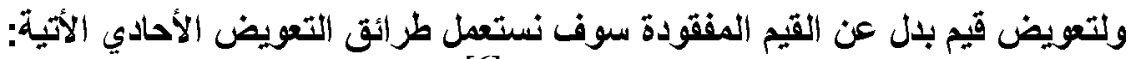

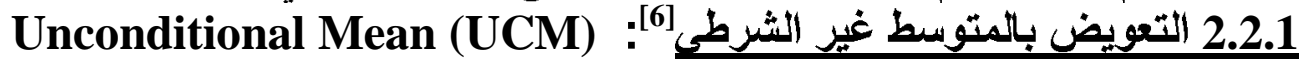

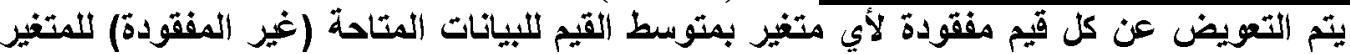

$$
\tilde{\mathrm{x}}_{\mathrm{j}}=\frac{\sum_{\mathrm{n}_{\mathrm{j}}} \mathrm{x}_{\mathrm{obs} .}}{\mathrm{n}_{\mathrm{j}}}
$$

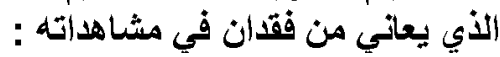

. $x_{j}$ : تمثثل عدد القيم المشاهدة فعلا للمتنغير

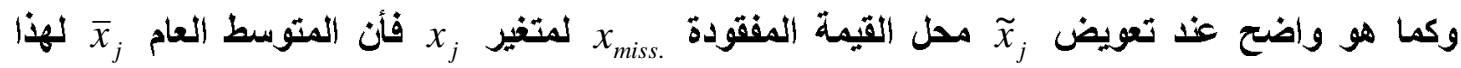

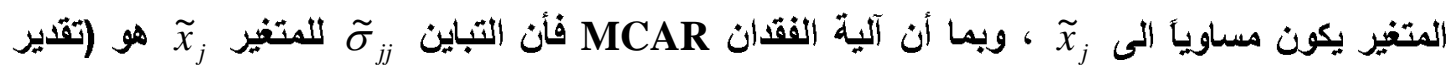

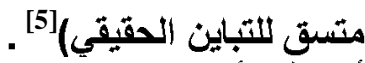

أن هذا الأسئوب يمكن استعماله في التعويض ولجميع أنماط الفقدان تحت شرط آلية فقدان MCAR.

Conditional Mean (CM) : 2.2.2 تعتمد هذا الطريقة على أستخدام أنموذج الانحدار الخطي، ولفهم آلية هذه الطريقة نفرض لائا متجه المتغيرات [

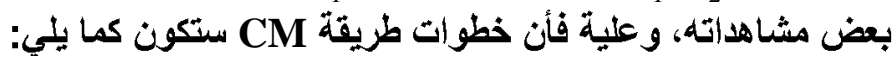

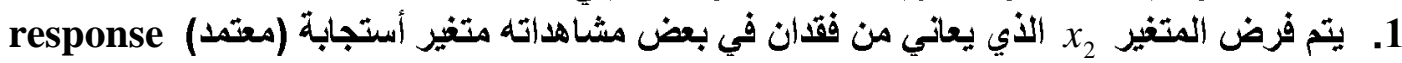
م variable للمتغير x x ، يصبح لاينا معادلة الانحدار الخطي التالية:

$$
\begin{aligned}
& \mathrm{x}_{\mathrm{i}^{\prime} 2}=\alpha+\beta \mathrm{x}_{\mathrm{i}^{\prime} 1}+\varepsilon_{\mathrm{i}^{\prime}} \\
& \mathrm{i}^{\prime}=1,2, \ldots . . \mathrm{n}_{\mathrm{c}} ; \mathrm{n}_{\mathrm{c}}<\mathrm{n}
\end{aligned}
$$

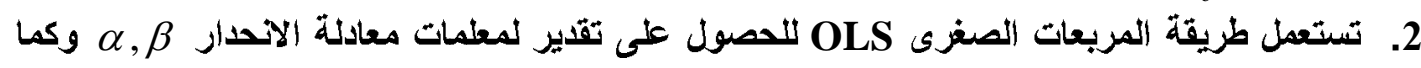

$$
\left(\begin{array}{l}
\hat{\alpha} \\
\hat{\beta}
\end{array}\right)=\left(X_{1}^{\prime} X_{1}\right)^{-1} X_{1}^{\prime} \underline{X}_{2}
$$

3. يتم حساب المتوسط الثرطي للمتغير x $x_{2}$ على المتغير x $x_{1}$ وكما في المعادلة الاتية وأن هذا المتوسط

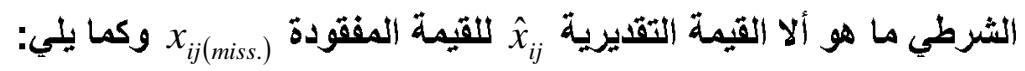

$$
\mathrm{E}\left(\mathrm{x}_{2} / \mathrm{x}_{1}\right)=\tilde{\mathrm{x}}_{2}+\tilde{\beta}\left(\mathrm{x}_{\mathrm{i} 1}-\tilde{\mathrm{x}}_{1}\right)
$$




$$
\left.\tilde{X}_{1}=\frac{\sum_{i^{\prime}=1}^{n_{c}} x_{i^{\prime} 1}}{n_{c}} \tilde{X}_{2}=\frac{\sum_{i^{\prime}=1}^{n_{c}} x_{i^{\prime} 2}}{n_{c}}\right\} \cdots(6)
$$

أن هذا الأسلوب في التعويض لا يمكن أستعمالة لجميع انماط الفقدان تحت شرط آلية فقدان MCAR

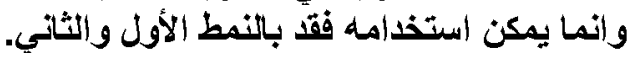

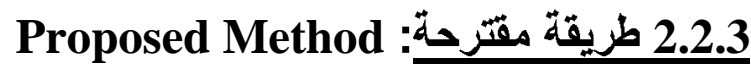

تستتد فكرة الطريقة على توليد قيم تحل محل القيم المفقودة وتتبع هذه القيم المولدة التوزيع الطبيعى

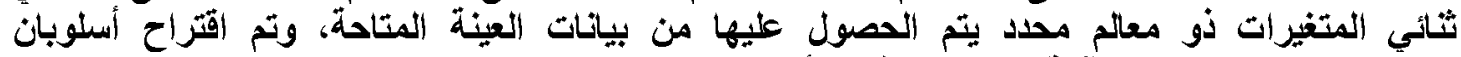

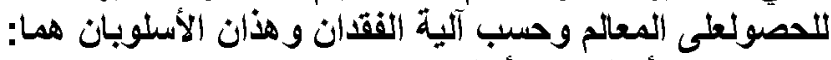

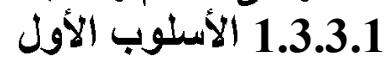

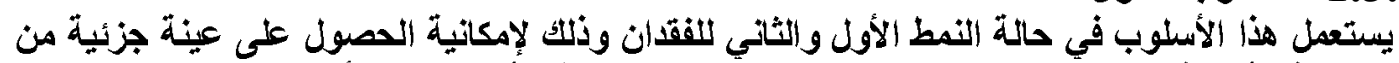

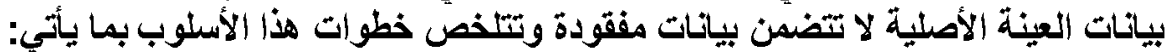

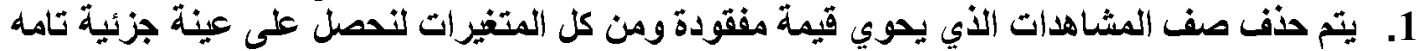
هن العينة الكلية .

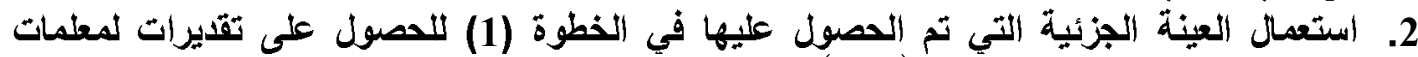

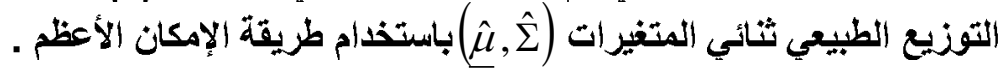

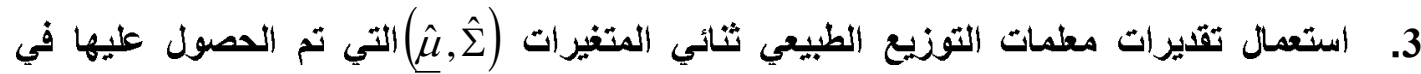

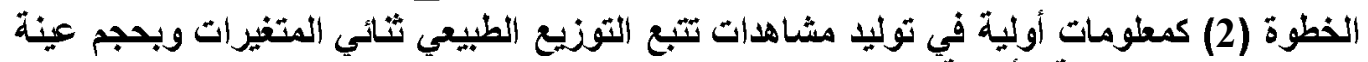

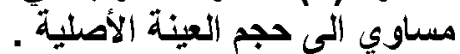
4. يتم مقارنة البيانات الأصلية مع البيانات المولدة باستخدام الخطوة (3) ويتم أحلال القيمة المولدة بلالا عن كل قيمة مفقودة تقابلها.

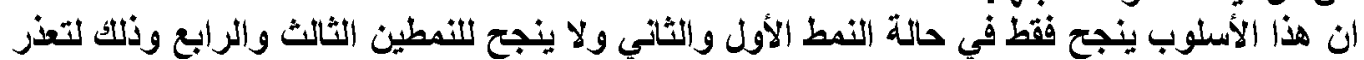

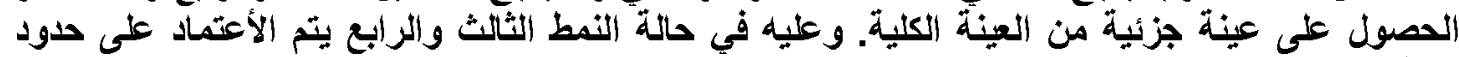

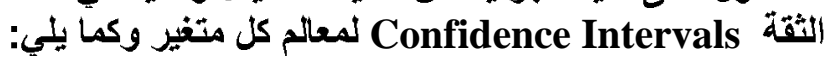




$$
\text { الطبيعي ثنائي المتغيرات }
$$

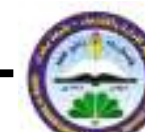
1.3.3.2 1 1 الأسلوب الثاني

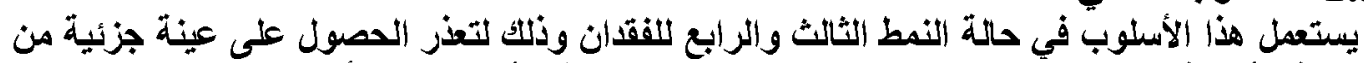

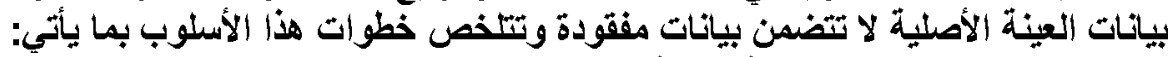

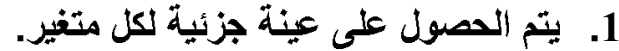
2. استعمال العينة الجزئية للحصول جلئية على تقليرات لمعلمات التوزيع الطبيعي ولكل متغيز باستخدام

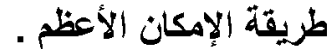
3. يتم الحصول على معلمات الإعم التوزيع الطبيعي ثنائي المتغيرات كما يأتي:

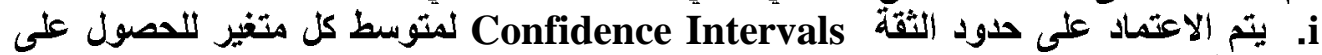
قيمة متجه المتوسطات .

$$
\bar{x}_{i} \pm t_{\left(\frac{\alpha}{2}, n_{i}-1\right)} \cdot \frac{s_{i}}{\sqrt{n}} \ldots(7)
$$

حيث أن من المشاهدات المتاحة لكل متغير

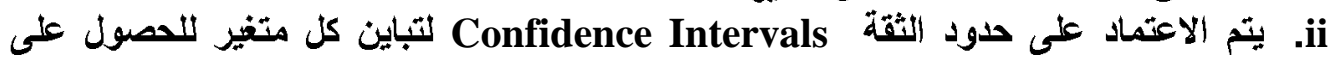

$$
\frac{(n-1) s_{i}^{2}}{\chi_{\frac{\alpha}{2}}^{2}} \leq \sigma_{i}^{2} \leq \frac{(n-1) s_{i}^{2}}{\chi_{\left(1-\frac{\alpha}{2}\right)}^{2}}
$$

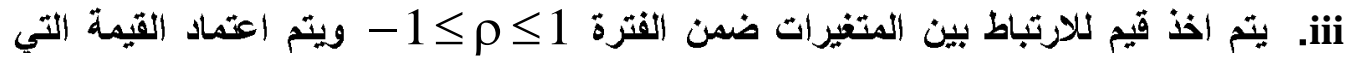

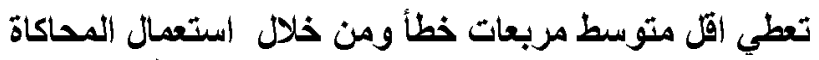

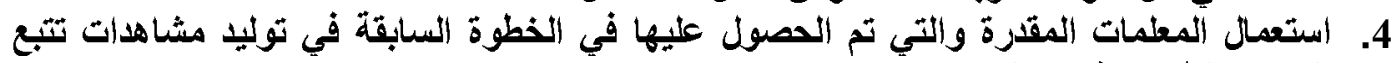

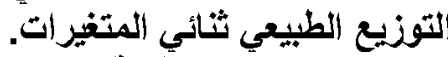

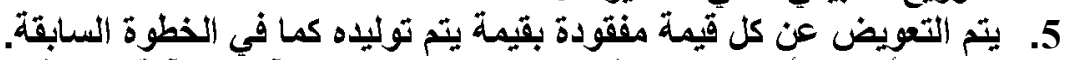

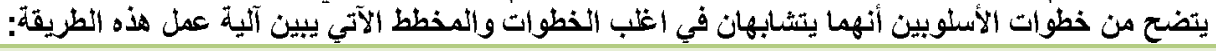

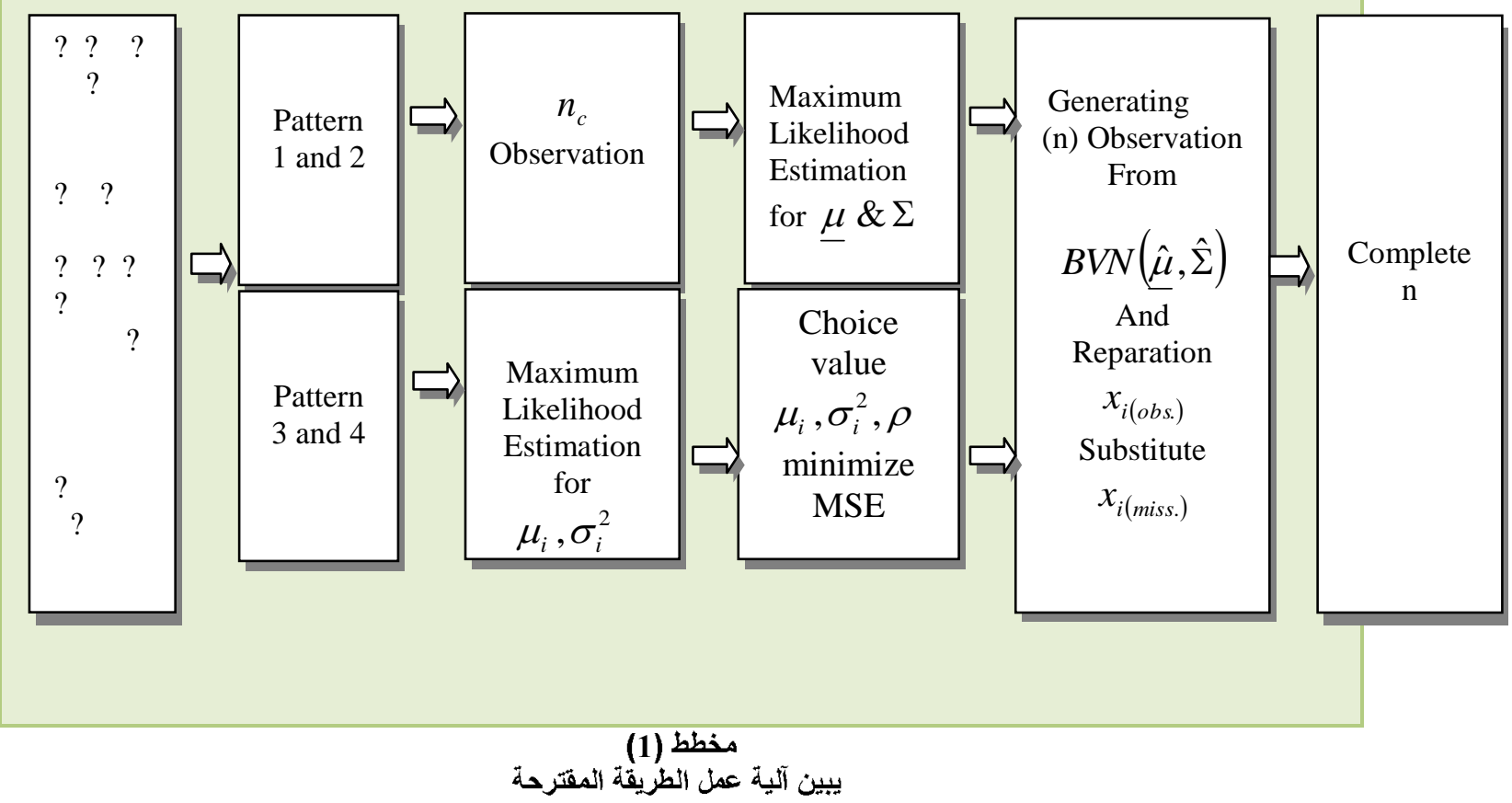




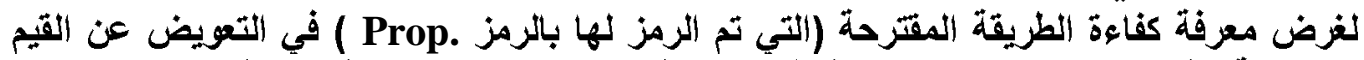

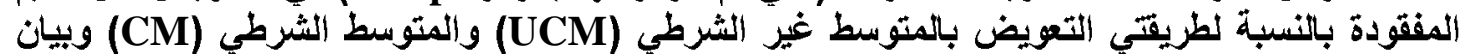

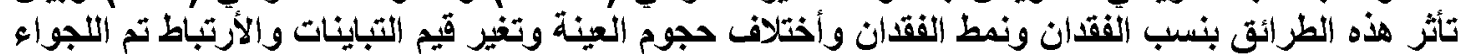

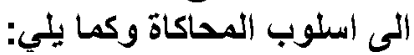

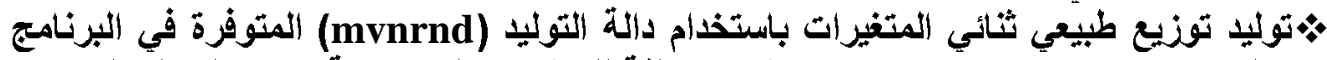

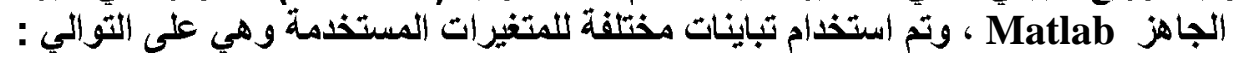

$$
\begin{aligned}
& X \sim N\left(\left(\begin{array}{l}
0 \\
0
\end{array}\right),\left(\begin{array}{ll}
1 & \rho \\
\rho & 1
\end{array}\right)\right) \\
& X \sim N\left(\left(\begin{array}{l}
0 \\
0
\end{array}\right),\left(\begin{array}{cc}
1 & \rho \sqrt{2} \\
\rho \sqrt{2} & 2
\end{array}\right)\right) \\
& X \sim N\left(\left(\begin{array}{l}
0 \\
0
\end{array}\right),\left(\begin{array}{cc}
5 & \rho \sqrt{15} \\
\rho \sqrt{15} & 3
\end{array}\right)\right)
\end{aligned}
$$

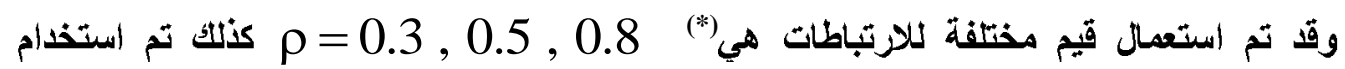
أحجام مختلفة للعينات

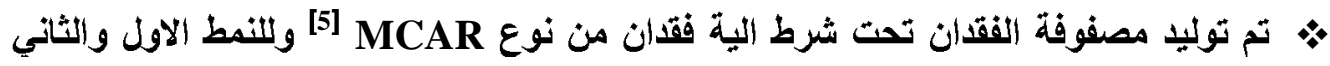

$$
\text { وينسب قة: }
$$

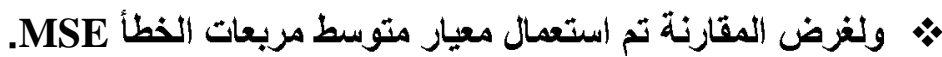

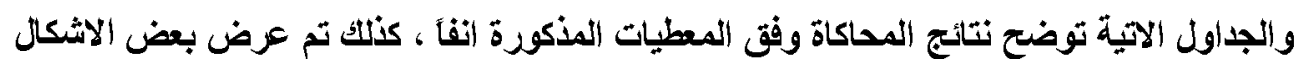
لعدد من الحالات المستخدمة. 
MSE( $\hat{f}(\underline{x}))$ يبين قيمة متوسط مربعات الخطأ لتقلير دالة الكثافة للتوزيع الطبيعي ثنائي المتغيرل

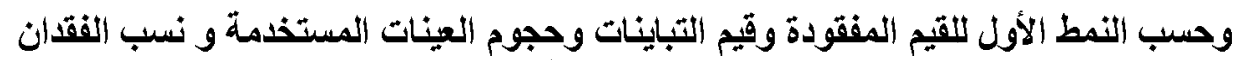

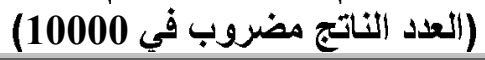

\begin{tabular}{|c|c|c|c|c|c|c|c|c|c|c|c|c|c|c|c|c|c|c|c|}
\hline \multicolumn{2}{|c|}{ Missing } & \multicolumn{9}{|c|}{$10 \%$} & \multicolumn{9}{|c|}{$20 \%$} \\
\hline \multirow{3}{*}{$n$} & $\rho$ & \multicolumn{3}{|c|}{0.3} & \multicolumn{3}{|c|}{0.5} & \multicolumn{3}{|c|}{0.8} & \multicolumn{3}{|c|}{0.3} & \multicolumn{3}{|c|}{0.5} & \multicolumn{3}{|c|}{0.8} \\
\hline & $\sigma_{1}^{2}$ & 1 & 1 & 5 & 1 & 1 & 5 & 1 & 1 & 5 & 1 & 1 & 5 & 1 & 1 & 5 & 1 & 1 & 5 \\
\hline & $\sigma_{2}^{2}$ & 1 & 2 & 3 & 1 & 2 & 3 & 1 & 2 & 3 & 1 & 2 & 3 & 1 & 2 & 3 & 1 & 2 & 3 \\
\hline \multirow{3}{*}{25} & $\hat{f}_{U C M}$ & 24 & 13 & 1.7 & 52 & 26 & 3.4 & 450 & 204 & 26 & 37 & 19 & 2.6 & 64 & 33 & 4.1 & 345 & 181 & 24 \\
\hline & $\hat{f}_{C M}$ & 32 & 17 & 2.1 & 83 & 41 & 5.5 & 1155 & 537 & 71 & 56 & 29 & 3.9 & 134 & 68 & 8.8 & 1861 & 901 & 120 \\
\hline & $\hat{f}_{\operatorname{Pr} o p}$ & 15 & 8 & 1.1 & 35 & 17 & 2.3 & 321 & 145 & 19 & 18 & 10 & 1.2 & 34 & 17 & 2.1 & 224 & 111 & 14 \\
\hline \multirow{3}{*}{50} & $\hat{f}_{U C M}$ & 12 & 6.1 & 1.6 & 27 & 14 & 1.8 & 237 & 137 & 19 & 21 & 11 & 1.4 & 39 & 19 & 2.5 & 224 & 110 & 15 \\
\hline & $\hat{f}_{C M}$ & 14 & 7.4 & 2.1 & 41 & 20 & 2.7 & 624 & 319 & 44 & 30 & 15 & 1.9 & 80 & 39 & 5.1 & 1105 & 572 & 77 \\
\hline & $\hat{f}_{\operatorname{Pr} o p}$ & 8 & 4.2 & 1.1 & 18 & 9.2 & 1.2 & 201 & 99 & 14 & 10 & 5.4 & 0.7 & 19 & 9.4 & 1.2 & 124 & 62 & 8.9 \\
\hline \multirow{3}{*}{100} & $\hat{f}_{U C M}$ & 8.3 & 4 & 0.5 & 18 & 9.8 & 1.3 & 224 & 113 & 15 & 15 & 7.6 & 0.9 & 29 & 14 & 1.9 & 179 & 91 & 12 \\
\hline & $\hat{f}_{C M}$ & 9.9 & 4.7 & 0.6 & 28 & 15 & 1.9 & 539 & 268 & 35 & 20 & 10 & 1.3 & 58 & 28 & 3.7 & 908 & 457 & 64 \\
\hline & $\hat{f}_{\operatorname{Pr} o p .}$ & 5.4 & 2.6 & 0.3 & 11 & 6.2 & 0.9 & 159 & 80 & 10 & 7.7 & 3.8 & 0.5 & 13 & 6.6 & 0.9 & 95 & 47 & 6.7 \\
\hline \multicolumn{2}{|c|}{ Missing } & \multicolumn{9}{|c|}{$30 \%$} & \multicolumn{9}{|c|}{$40 \%$} \\
\hline \multirow{3}{*}{$n$} & $\rho$ & \multicolumn{3}{|c|}{0.3} & \multicolumn{3}{|c|}{0.5} & \multicolumn{3}{|c|}{0.8} & \multicolumn{3}{|c|}{0.3} & \multicolumn{3}{|c|}{0.5} & \multicolumn{3}{|c|}{0.8} \\
\hline & $\sigma_{1}^{2}$ & 1 & 1 & 5 & 1 & 1 & 5 & 1 & 1 & 5 & 1 & 1 & 5 & 1 & 1 & 5 & 1 & 1 & 5 \\
\hline & $\sigma_{2}^{2}$ & 1 & 2 & 3 & 1 & 2 & 3 & 1 & 2 & 3 & 1 & 2 & 3 & 1 & 2 & 3 & 1 & 2 & 3 \\
\hline \multirow{3}{*}{25} & $\hat{f}_{U C M}$ & 61 & 30 & 3.8 & 86 & 48 & 6.1 & 307 & 155 & 20 & 82 & 44 & 5 & 112 & 59 & 8 & 302 & 146 & 18 \\
\hline & $\hat{f}_{C M}$ & 117 & 56 & 7.5 & 279 & 154 & 20 & 3939 & 1895 & 242 & 207 & 120 & 15 & 567 & 333 & 32 & 7644 & 3451 & 522 \\
\hline & $\hat{f}_{\operatorname{Pr} o p}$ & 20 & 11 & 1.3 & 33 & 15 & 2.1 & 140 & 72 & 9.3 & 22 & 11 & 1 & 33 & 17 & 2 & 110 & 56 & 7 \\
\hline \multirow{3}{*}{50} & $\hat{f}_{U C M}$ & 32 & 16 & 2.4 & 55 & 27 & 3.5 & 201 & 95 & 13 & 54 & 26 & 3.6 & 72 & 36 & 4.8 & 185 & 95 & 12 \\
\hline & $\hat{f}_{C M}$ & 51 & 26 & 3.8 & 152 & 73 & 9.5 & 2143 & 977 & 134 & 104 & 48 & 7.8 & 270 & 234 & 18 & 3921 & 1886 & 255 \\
\hline & $\hat{f}_{\operatorname{Pr} o p}$ & 12 & 6.3 & 0.9 & 20 & 10 & 1.3 & 90 & 41 & 6 & 15 & 7.4 & 0.9 & 21 & 11 & 1.4 & 70 & 36 & 4.6 \\
\hline \multirow{3}{*}{100} & $\hat{f}_{U C M}$ & 26 & 13 & 1.7 & 40 & 21 & 3 & 159 & 80 & 10 & 42 & 21 & 2.8 & 57 & 21 & 2.7 & 142 & 74 & 9.6 \\
\hline & $\hat{f}_{C M}$ & 39 & 19 & 2.5 & 103 & 53 & 7 & 1605 & 810 & 107 & 73 & 36 & 4.9 & 193 & 35 & 4.6 & 2881 & 1411 & 190 \\
\hline & $\hat{f}_{\operatorname{Pr} o p}$ & 10 & 5 & 0.7 & 15 & 8 & 1 & 68 & 34 & 5 & 13 & 6.2 & 0.8 & 17 & 6.2 & 0.8 & 52 & 27 & 3.6 \\
\hline
\end{tabular}




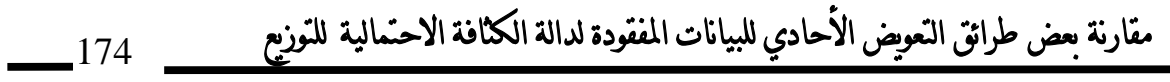

$$
\text { الطبيعي ثنائن المتغرات }
$$

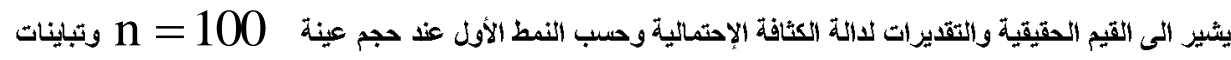

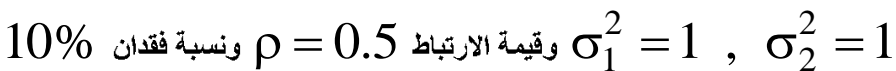
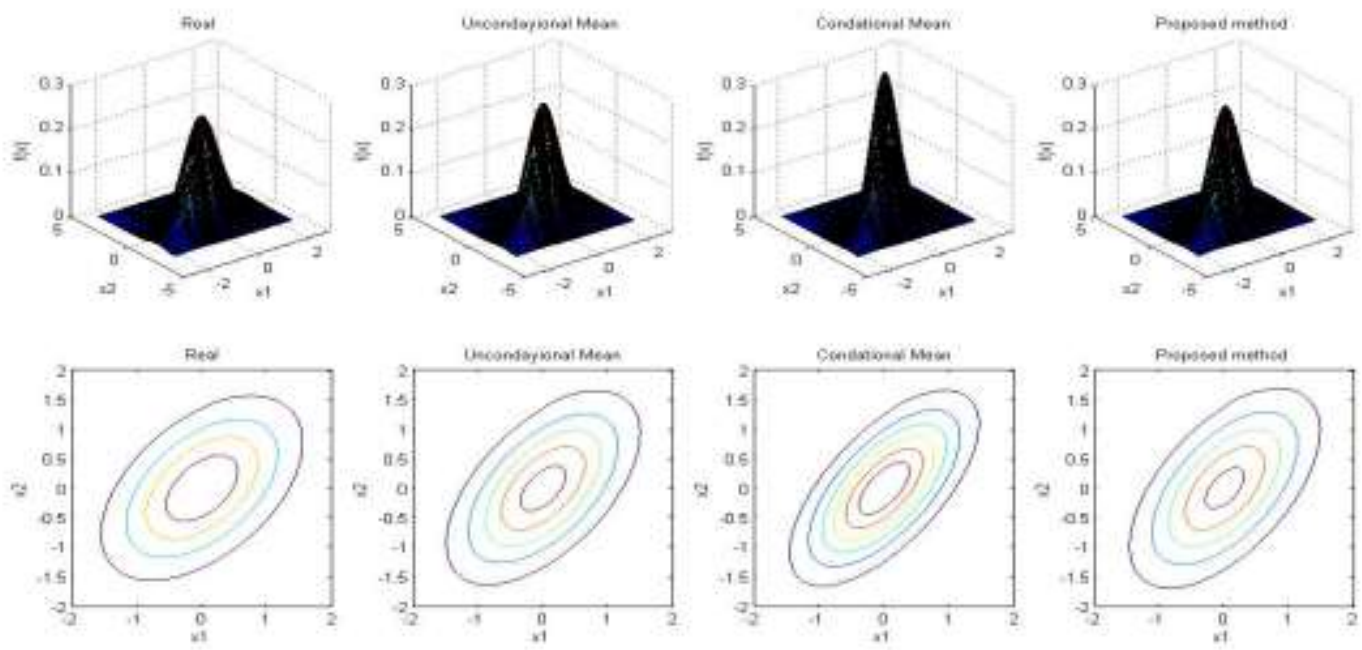

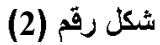

يشير اللى القيم الحقيقية والتقليرات لدالة الكثافة الإحتمالية وحسب النمط الأول عند حجم عينة n = 100 وتباينات $20 \%$ o $0.5=1, \sigma_{2}^{2}=1$
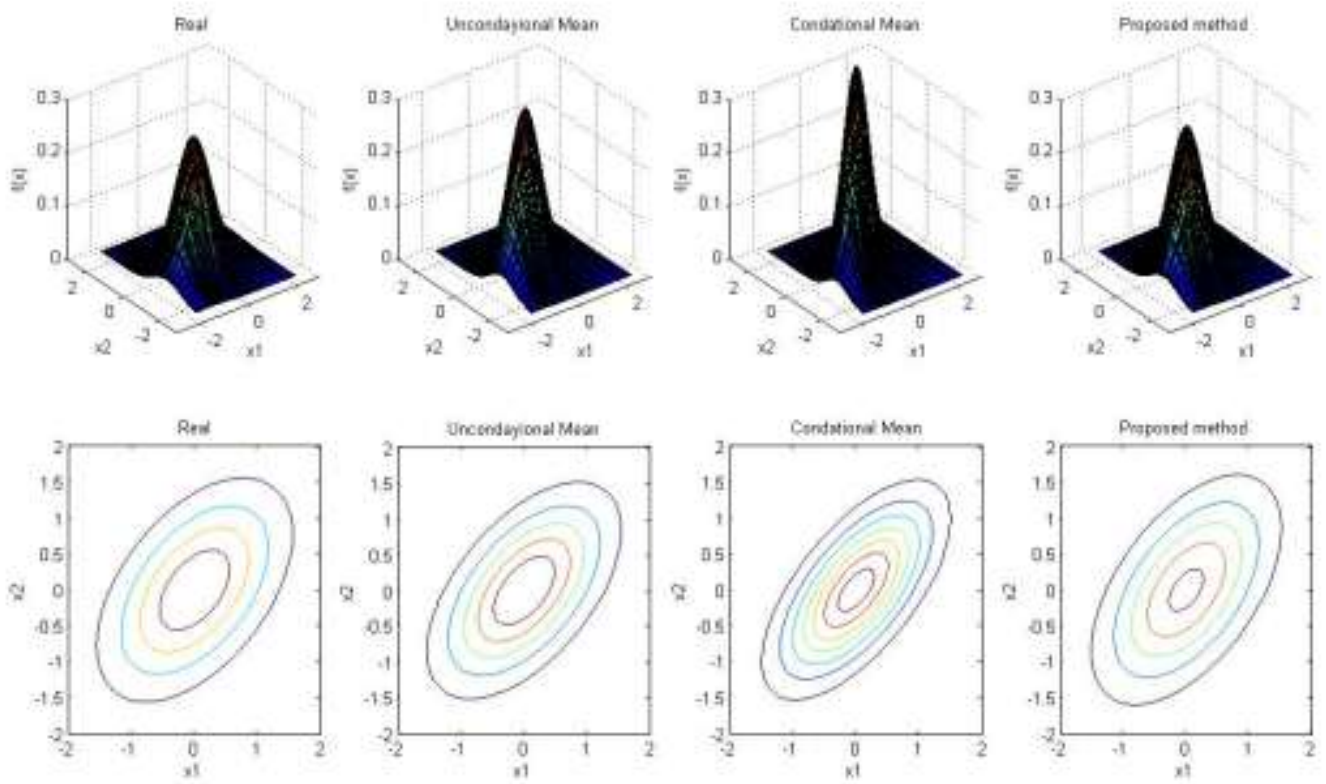


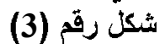

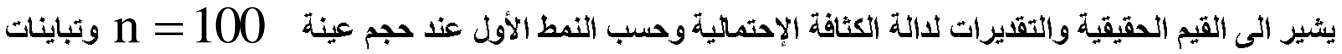

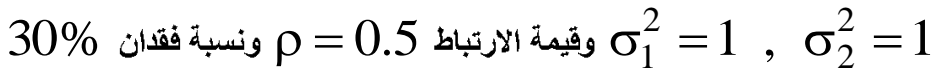
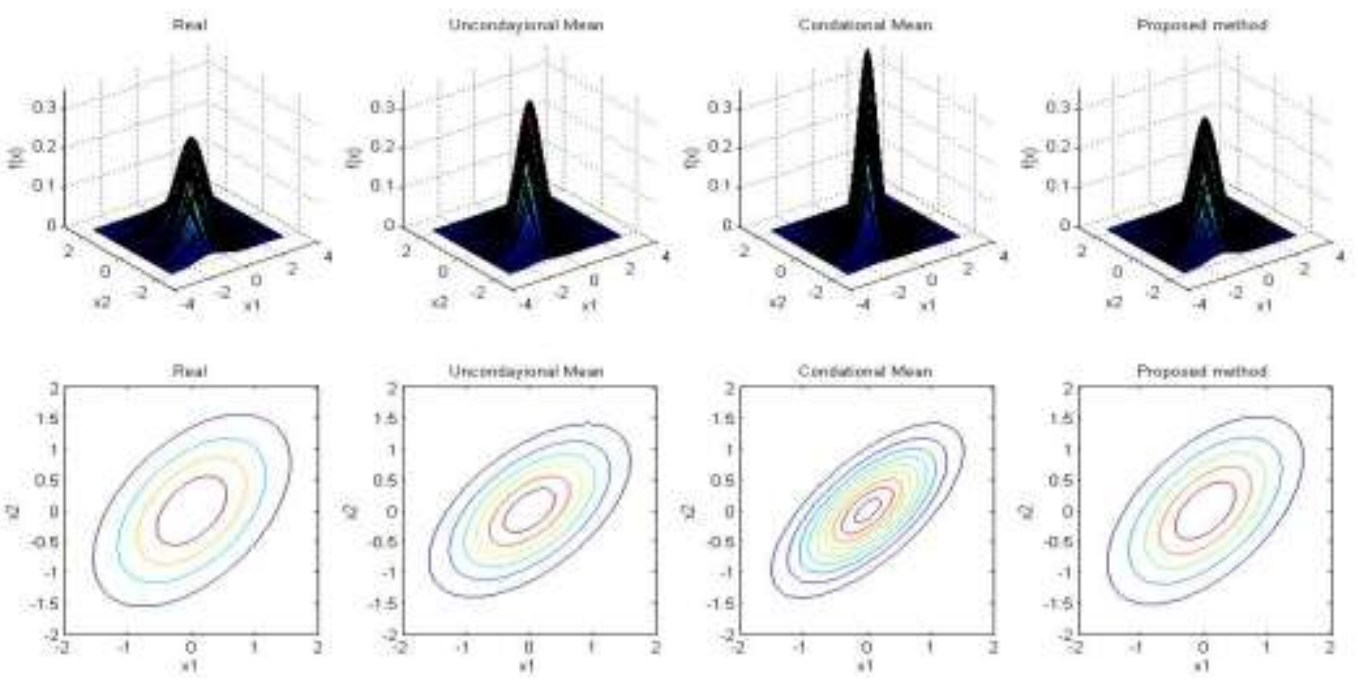

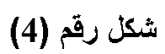

يثير الى القيم الحقيقية والتقيرات لاالة أكثافة الإحتمالية وحسب النمط الأول عثد حجم عينة 40\% $\sigma_{1}^{2}=1, \sigma_{2}^{2}=1$
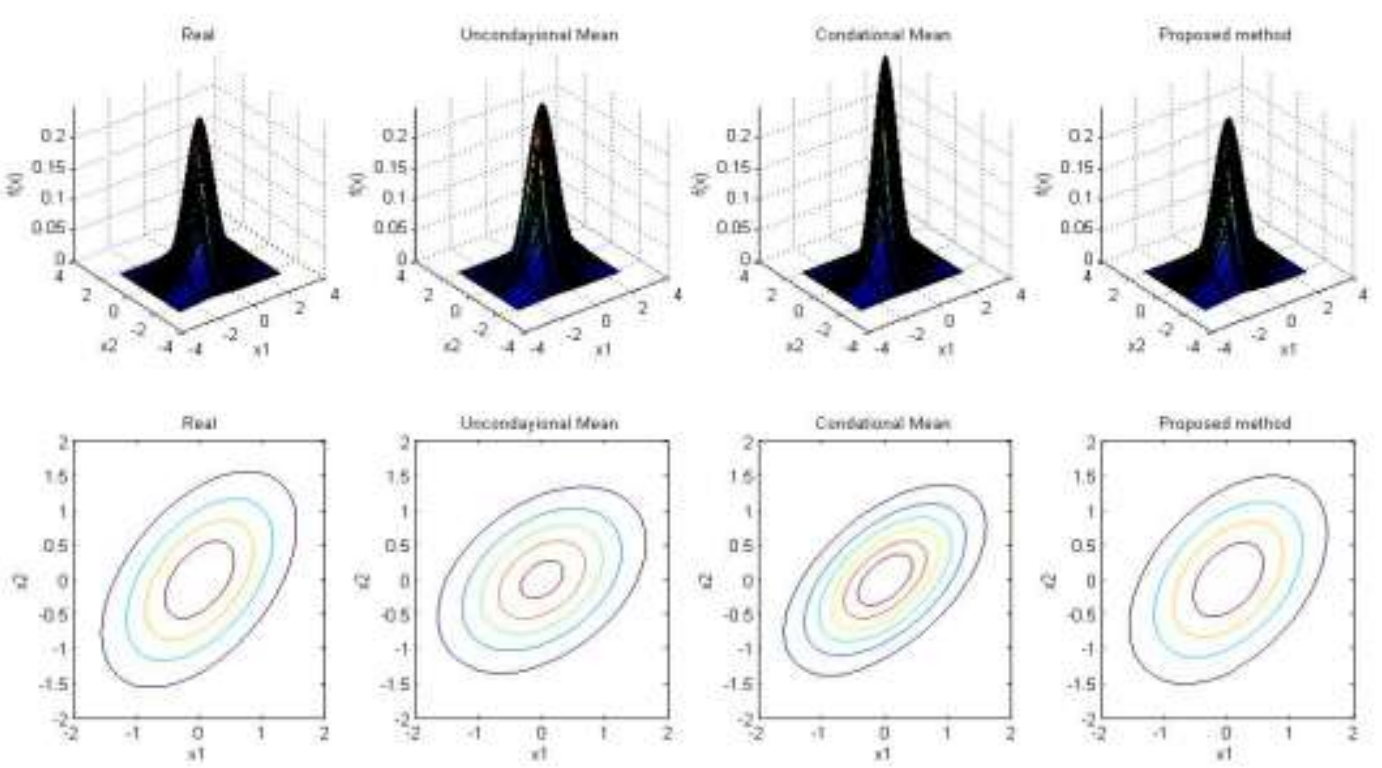


\section{الطبيعي ثنائي المتغيرات}

\section{جلدول (2)}

يبين قيمة متوسط مربعات الخطأ لتقاير دالة الكثافة للتوزيع الطبيعي ثنائي المتفيرات (2) للقيم المفقودة وقيم التباينات وحجوم العينات المستخدمة و نسب الفقدان

(العدد الناتج مضروب في التئ 10000)

\begin{tabular}{|c|c|c|c|c|c|c|c|c|c|c|c|c|c|c|c|c|c|c|c|}
\hline \multicolumn{2}{|c|}{ Missing } & \multicolumn{9}{|c|}{$10 \%$} & \multicolumn{9}{|c|}{$20 \%$} \\
\hline \multirow{3}{*}{$n$} & $\rho$ & \multicolumn{3}{|c|}{0.3} & \multicolumn{3}{|c|}{0.5} & \multicolumn{3}{|c|}{0.8} & \multicolumn{3}{|c|}{0.3} & \multicolumn{3}{|c|}{0.5} & \multicolumn{3}{|c|}{0.8} \\
\hline & $\sigma_{1}^{2}$ & 1 & 1 & 5 & 1 & 1 & 5 & 1 & 1 & 5 & 1 & 1 & 5 & 1 & 1 & 5 & 1 & 1 & 5 \\
\hline & $\sigma_{2}^{2}$ & 1 & 2 & 3 & 1 & 2 & 3 & 1 & 2 & 3 & 1 & 2 & 3 & 1 & 2 & 3 & 1 & 2 & 3 \\
\hline \multirow{2}{*}{25} & $\hat{f}_{U C M}$ & 26 & 12 & 1.7 & 40 & 19 & 2.6 & 185 & 100 & 13 & 42 & 19 & 2.7 & 59 & 27 & 4.1 & 192 & 99 & 13 \\
\hline & $\hat{f}_{\text {Prop. }}$ & 34 & 19 & 5.2 & 43 & 25 & 8.9 & 110 & 59 & 30 & 36 & 20 & 5.8 & 34 & 26 & 8.2 & 103 & 53 & 19 \\
\hline \multirow{2}{*}{50} & $\hat{f}_{U C M}$ & 14 & 7.2 & 9.9 & 24 & 12 & 1.7 & 146 & 66 & 9.6 & 27 & 14 & 1.9 & 38 & 21 & 2.6 & 127 & 63 & 8.7 \\
\hline & $\hat{f}_{\operatorname{Pr} o p}$ & 28 & 15 & 4.6 & 34 & 20 & 7 & 87 & 46 & 23 & 30 & 17 & 4.7 & 35 & 21 & 6.3 & 83 & 41 & 14 \\
\hline \multirow{2}{*}{100} & $\hat{f}_{U C M}$ & 11 & 5.5 & 7.2 & 20 & 10 & 1.3 & 124 & 62 & 8 & 21 & 11 & 1.4 & 32 & 16 & 2.1 & 107 & 53 & 7.1 \\
\hline & $\hat{f}_{\operatorname{Pr} o p}$ & 26 & 14 & 4.2 & 31 & 18 & 6.4 & 76 & 39 & 20 & 27 & 15 & 4.2 & 32 & 19 & 5.6 & 73 & 36 & 11 \\
\hline \multicolumn{2}{|c|}{ Missing } & \multicolumn{9}{|c|}{$30 \%$} & \multicolumn{9}{|c|}{$40 \%$} \\
\hline \multirow{3}{*}{$n$} & $\rho$ & \multicolumn{3}{|c|}{0.3} & \multicolumn{3}{|c|}{0.5} & \multicolumn{3}{|c|}{0.8} & \multicolumn{3}{|c|}{0.3} & \multicolumn{3}{|c|}{0.5} & \multicolumn{3}{|c|}{0.8} \\
\hline & $\sigma_{1}^{2}$ & 1 & 1 & 5 & 1 & 1 & 5 & 1 & 1 & 5 & 1 & 1 & 5 & 1 & 1 & 5 & 1 & 1 & 5 \\
\hline & $\sigma_{2}^{2}$ & 1 & 2 & 3 & 1 & 2 & 3 & 1 & 2 & 3 & 1 & 2 & 3 & 1 & 2 & 3 & 1 & 2 & 3 \\
\hline \multirow{2}{*}{25} & $\hat{f}_{U C M}$ & 67 & 34 & 4.7 & 76 & 40 & 5.4 & 168 & 86 & 13 & 77 & 39 & 5.3 & 96 & 47 & 6.6 & 165 & 89 & 11 \\
\hline & $\hat{f}_{\text {Prop. }}$ & 38 & 23 & 6.5 & 45 & 27 & 7.6 & 99 & 50 & 14 & 39 & 25 & 6.8 & 46 & 28 & 8.3 & 96 & 48 & 12 \\
\hline \multirow{2}{*}{50} & $\hat{f}_{U C M}$ & 41 & 21 & 2.8 & 55 & 27 & 3.6 & 121 & 57 & 7.6 & 53 & 27 & 3.5 & 65 & 32 & 4.2 & 112 & 56 & 7.7 \\
\hline & $\hat{f}_{\text {Prop. }}$ & 31 & 18 & 5.2 & 36 & 21 & 6.3 & 81 & 39 & 10 & 33 & 21 & 5.8 & 37 & 23 & 6.5 & 78 & 38 & 9.3 \\
\hline \multirow{2}{*}{100} & $\hat{f}_{U C M}$ & 34 & 17 & 2.3 & 43 & 21 & 2.9 & 97 & 45 & 6.4 & 41 & 21 & 2.7 & 49 & 25 & 3.3 & 88 & 44 & 6 \\
\hline & $\hat{f}_{\text {Prop. }}$ & 28 & 17 & 4.8 & 33 & 19 & 5.7 & 73 & 35 & 8.8 & 29 & 19 & 5.3 & 33 & 21 & 6 & 71 & 35 & 8 \\
\hline
\end{tabular}




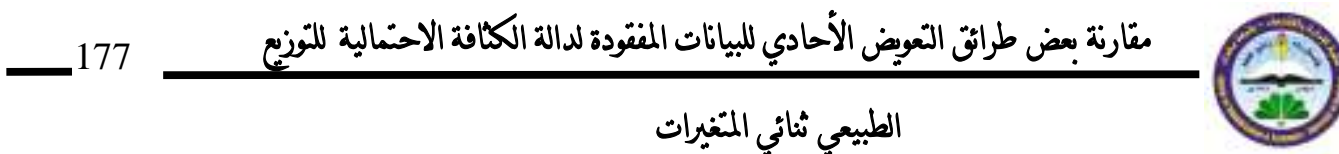

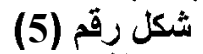

يشير الى القيم الحقيقية والتقيرات لدالة الكثافة الإحتمالية وحسب النمط الثالث عثد حجم عينة 10\% و 100
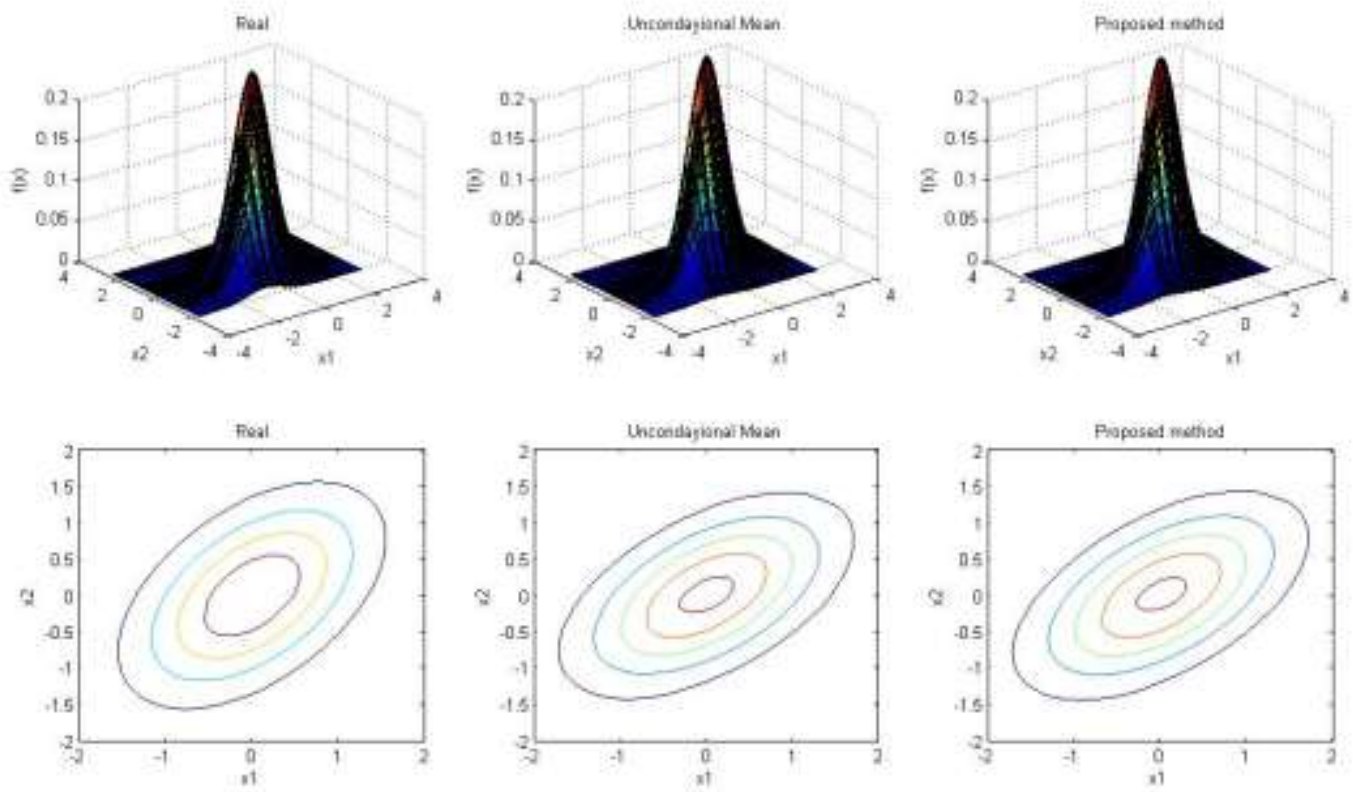

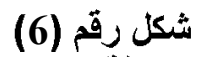

يشير الى القيم الحقيقية والتقيرات لادالة الكثافة الإحتمالية وحسب النمط الثالث عثد حجم عينة

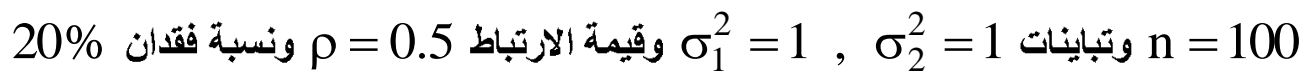
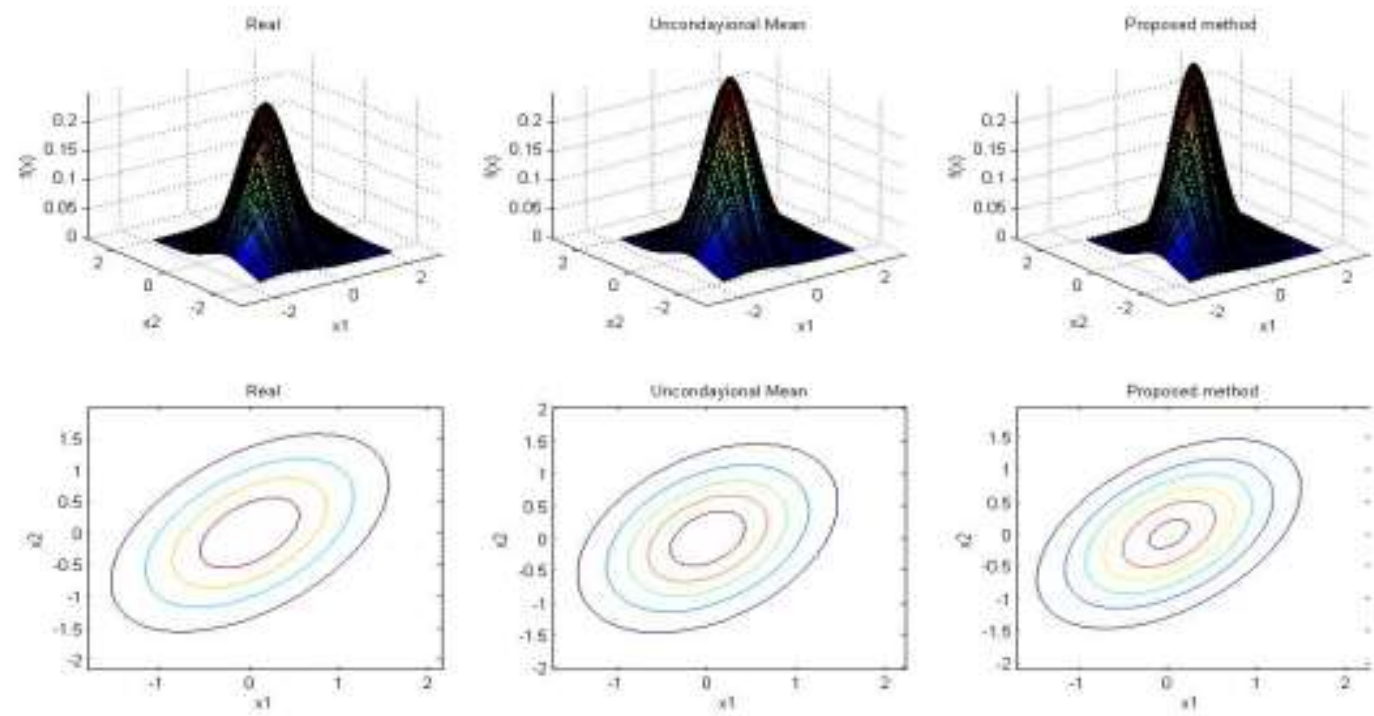


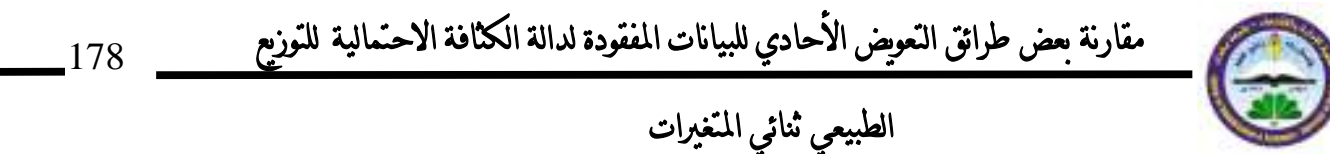

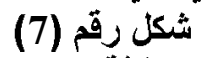

يشير الى القيم الحقيقية والتقديرات لدالة الكثافة الإحتمالية وحسب النمط الثالث عند حجم عينة 30\% و 30 = 100
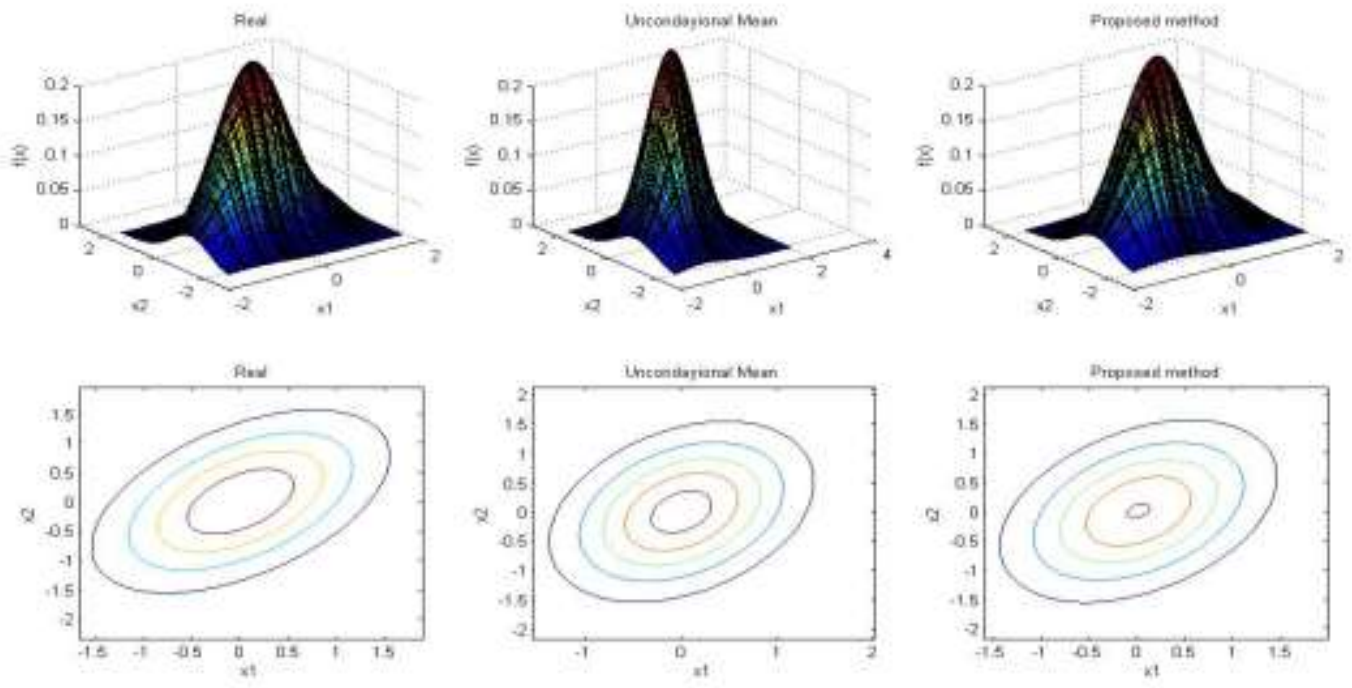

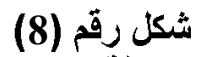

يثير اللى القيم الحقيقية والتقليرات لادلة الكثافة الإحتمالية وحسب النمط الثالث عث حجم عينة 40\% و 0 = 100
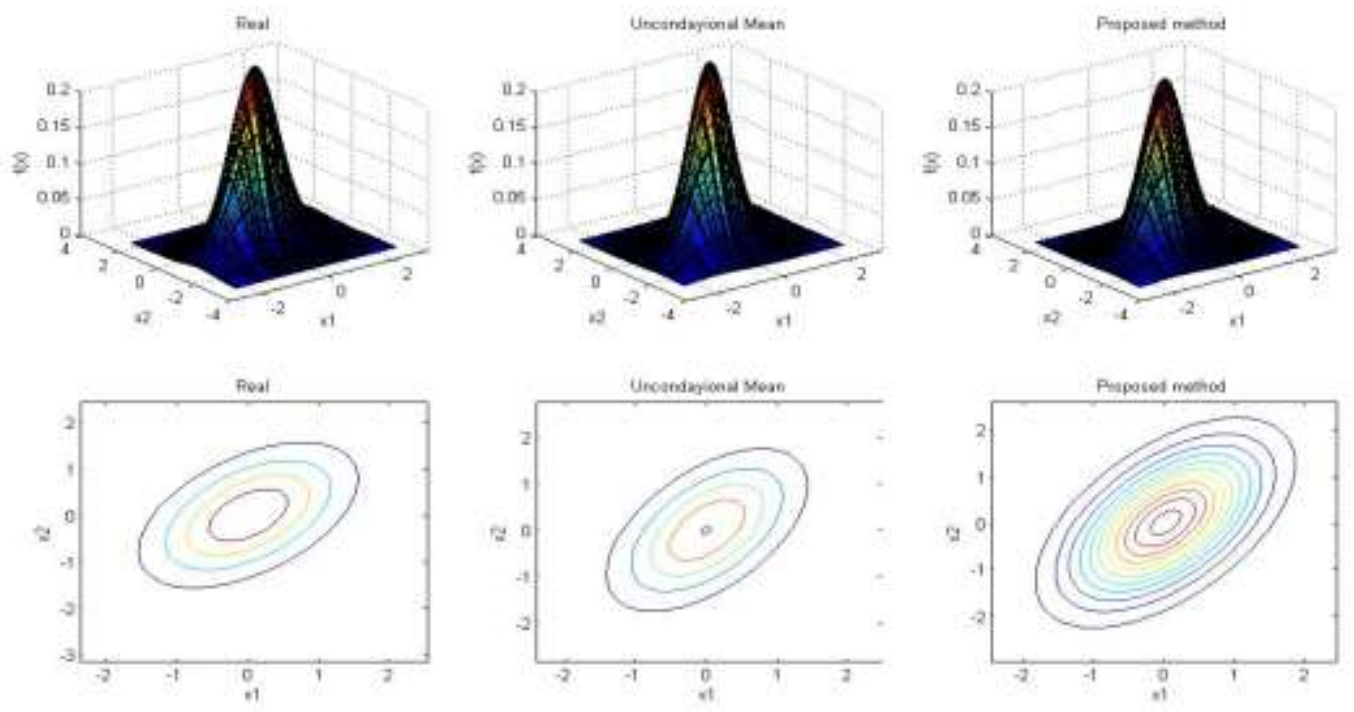
الطبيعي ثنائي المتغيرات

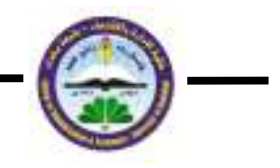

4. تفسير النتشائج

من الجدول (1) نلاحظ النتائج الاتية: (النمط الأول للفقان انجان

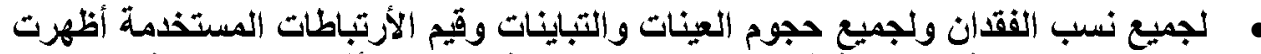

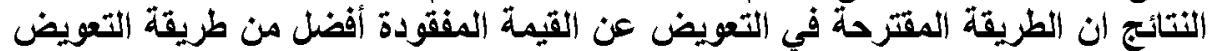

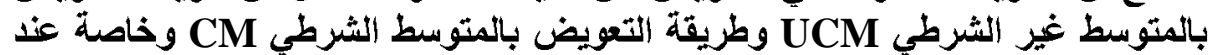

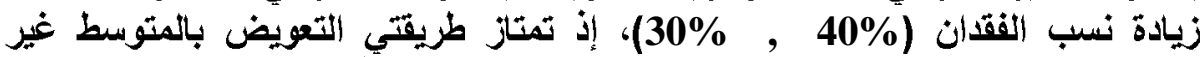

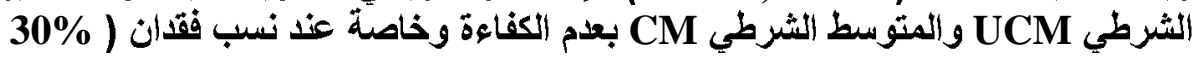

نلاحظ عذ زيادة قيم الارتباطات استقرار الطريقة المثترحة ولكن نلاحظ تأثر ويشكل كبير

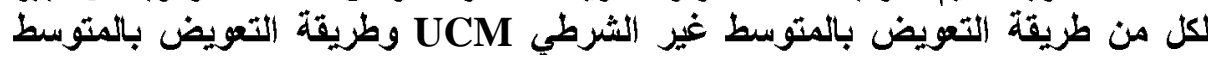

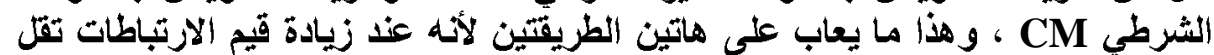
كفاءة هاتين الطريفتين.

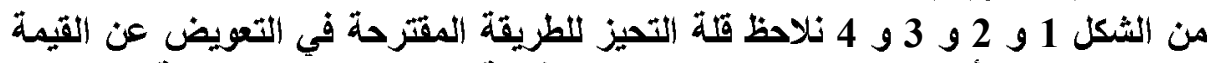

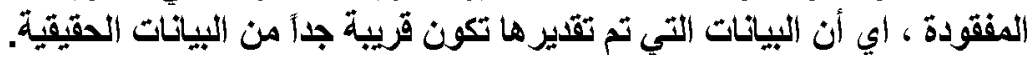

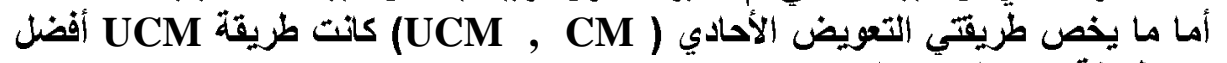

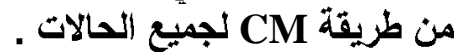

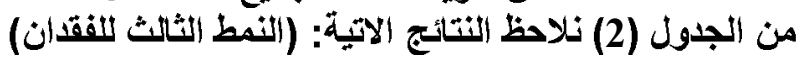

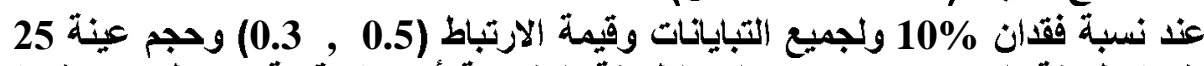

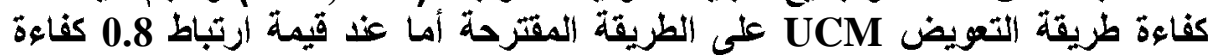
الطريقة المقترحة عند قيمة تباين حجم عينة 50 و 100 نلاحظ كفاءة الطريقة UCM عند قيمة ارتباط 0.5 ولكن عند قيمة

$$
\text { أرتباط } 0.3 \text { تكون الطريقة المقترحة أفضل عندما }
$$

• عد نسبة فقدان $20 \%$ وقيمة ارتباط (0.5 , 0.3) ولجميع حجوم العينات تكون قيم

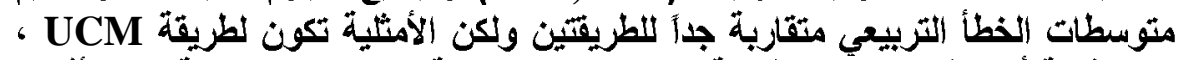

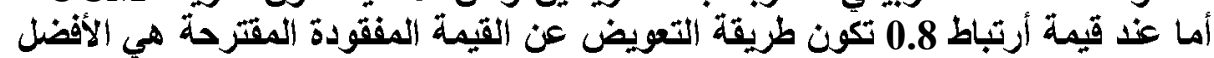

$$
\text { وخاصة عندما }
$$

• عد نسبة فقدان (30\% , 40\%) ولجميع حجوم العينات وقيم الارتباطات وعندما تكون الطريقة المقترحة في التعويض عن القيمة $\underline{\sigma}^{2}=\left(\begin{array}{l}1 \\ 1\end{array}\right), \underline{\sigma}^{2}=\left(\begin{array}{l}1 \\ 2\end{array}\right)$ المفقودة هي الأفضل.

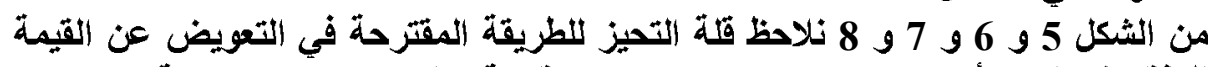

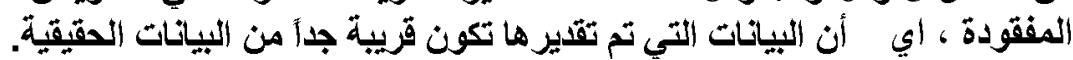




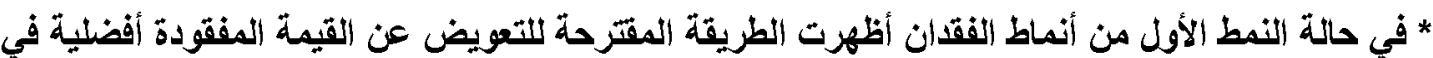

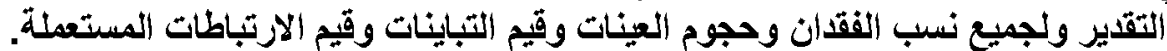

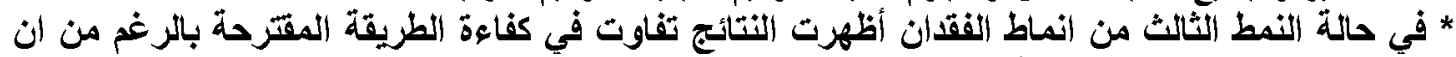

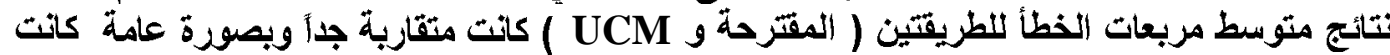

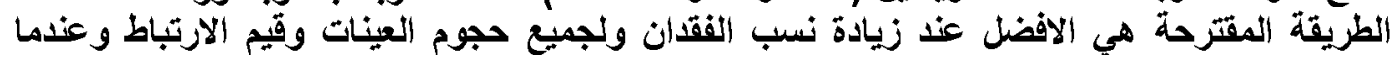

$$
\cdot \underline{\sigma}^{2}=\left(\begin{array}{l}
1 \\
1
\end{array}\right), \underline{\sigma}^{2}=\left(\begin{array}{l}
1 \\
2
\end{array}\right)
$$

6

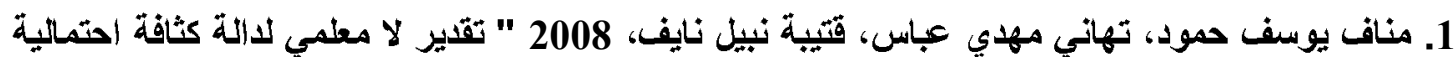

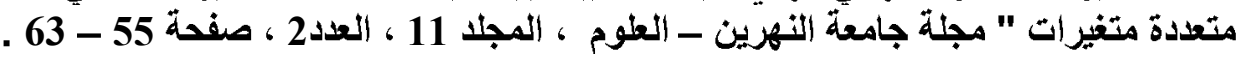
2. Dagenais, M.G. (1973) "The use of Incomplete Observation in Multiple Regression Analysis: A generalized Least Squares Approach" J. of Econometrics, vol. 1 , p. 317 - 328.

3. Heumann, C.; Nittner, T.; Scheid, S. \& Toutenburg, H. (2002)"Parametric and Nonparametric Regression Missing X's: A Review" www. Stat.uni-muenchen.de /sfb386/papers/dsp/paper286.ps.

4. Kosobud, R. (1963) "A note on Problem Caused by Assignment of Missing Data in Sample Surveys" Econometric a, vol. 31, p. 562 - 563.

5. Little, R.J.A \& Rubin, D.B (2003) "Statistical Analysis with Missing Data" $2^{\text {nd }}$ ed., John Wile \& Sons, New York.

6. Nittner, T. (2002) "The Additive with Missing values in the Independent Variable: Theory \& Simulation" http://www.pms.ifi.Imu.de/researchreport/index.pdf 